\title{
Filter Design for Suppressing Sidelobe Interference in NC- OFDM System
}

\author{
Haojie Yuan ${ }^{a}$, Erlei Li ${ }^{b}$, Fanchang Zeng $^{\mathrm{c}}$ \\ Jiangsu Automation Research Institute, Lianyungang, Jiangsu, 222000, China. \\ azeroyuan814@163.com, b17826531077@163.com, cZengfanchang0112@163.com
}

\begin{abstract}
OFDM technology plays an important role in multicarrier modulation. Combining with cognitive radio technology, the traditional OFDM transform into NC-OFDM. The two main drawback of the NC-OFDM system are the secondary users' sidelobe interference to primary users and the high PAPR (peak to average power ratio). One way to suppress sidelobe interference of primary users is to design the filter in the time domain and the PTS method can effectively reduce the PAPR of the NC-OFDM system. According to the distribution of subcarriers in NC-OFDM system, this paper proposes a model of secondary user's interference to primary users. The average interference power density is defined for this model, which is used to measure the interference level of different filters applied in NC-OFDM system. After filtering in time domain, it will cause PAPR to pick up. In this paper, we still use the PTS method to suppress PAPR, and can obtain better system performance.
\end{abstract}

Keywords: filter design, NC-OFDM, sidelobe interference, PAPR, PTS.

\section{Introduction}

With the rapid development of wireless communication technology, the needs for wireless spectrum resources is increasing, and current spectrum resources have become increasingly lacking. Cognitive Radio (CR) can realize opportunistic communication without disturbing the primary user (PU) because it can dynamically detect the idle time and frequency band that is not occupied in real time and adjust the parameters of the transmitter accordingly [1]. Therefore, CR is one of the effective means to solve the current lack of radio spectrum. Orthogonal frequency division multiplexing (OFDM) can provide high-speed communication, at the same time, it can let radio channel interference against multi-channel with low cost, and it is widely used in the wireless communication standard [2]. The non-continuous orthogonal frequency division multiplexing (NC-OFDM) is improved based on the conventional OFDM, and the subcarriers corresponding to the PU in the active state are turned off, and the user is informed to communicate using the discontinuous idle subcarriers. To avoid the interference to PU, we should minimize the relative interference power of the secondary users (SU) to the primary users to avoid the special location of the users.

In References [3, 4], it is suggested that the interference of the SU to the PU is the main interference in the NC-OFDM system, and it is suggested that using time domain filter design technique can reduce the interference. The use of Dolph-Chebyshev window in Reference [4] effectively reduces the SU's interference to PU, but the disadvantage is sacrificing the certain subcarrier orthogonality. Although the use of pretreatment technology can improve this phenomenon but reduce the spectral efficiency. In actual cognitive radio system, since the location of the PU in the active state is random, and its spectrum occupancy rate may change with time. To ensure the orthogonal characteristics of subcarriers in NC-OFDM system, five different types of Nyquist windows were selected. To suppress the sidelobe interference of SU to PU, five kinds of filter functions are applied to NC-OFDM system. And use the average relative interference power density function to measure the filter function to suppress sidelobe interference performance. The SOCW window with the best performance is selected.

NC-OFDM signals are overlaped by multiple subcarrier signals, resulting in a very high PAPR. It will increase the linear amplification requirements of the linear power amplifier, which will greatly reduce the efficiency of the RF power amplifier. In his paper,we use PTS method to suppress the PAPR. 


\section{Organization of the Text}

\subsection{Analysis of Filter Model in NC-OFDM System.}

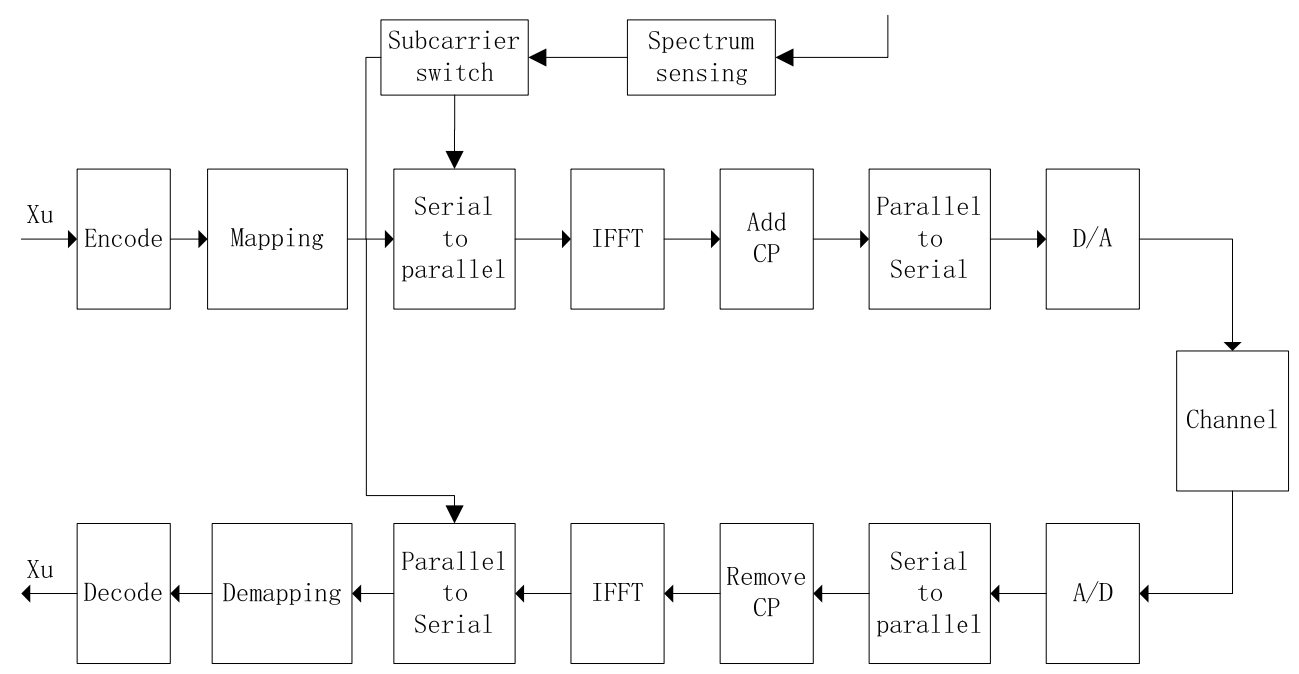

Fig. 1 The System Block Diagram of NC-OFDM

As shown in Figure 1, NC-OFDM system allocates subcarriers by sensing external spectral information. The subcarriers that corresponding to primary users is set to 0 , making them become unuseful subcarriers. The modulation data is allocated on the subcarriers that corresponding to cognitive users. Thus, the transmitter of NC-OFDM system is formed. Assuming that the number of subcarriers for cognitive users in NC-OFDM system are $N_{u}$, and the total number of subcarriers are $N$, the baseband signal after IFFT (inverse fast Fourier transform) is [6]:

$$
x(n)=\frac{1}{\sqrt{N}} \sum_{k=0}^{N-1} X_{k} e^{j 2 \pi k n / N}
$$

Where $1 / \sqrt{N}$ represents power normalization factor, and $0 \leq n \leq N-1 ; X_{k}$ denotes the modulation signals allocated to the subcarrier $k$. When $k$ is primary subcarrier, $X_{k}=0$;

Figure 2 shows the distribution of subcarriers in the NC-OFDM system. In the figure, the primary users and the secondary users share the spectrum resource. The secondary users correspond to the valid data subcarriers. The primary users correspond to the invalid subcarriers. There will be a corresponding protection bandwidth between the primary users and the secondary users. In order to reduce the use of guard bandwidth, the effective sub-carriers should be placed in a continuous segment as much as possible.

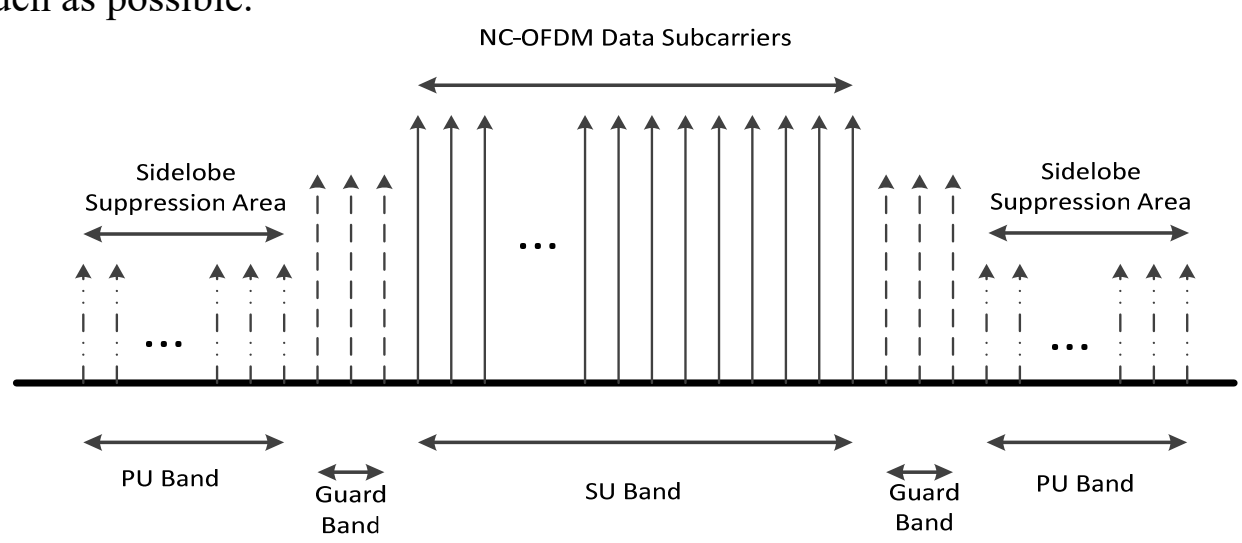

Fig.2 Allocation of Subcarriers in NC-OFDM System 
Figure 3 shows the time-domain NC-OFDM symbol obtained after adding the filter. $N_{w}$ in Figure 2 is for the rolling overlap part; $N_{g}$ is for the protection interval; $N$ is for the IFFT effective integration area.

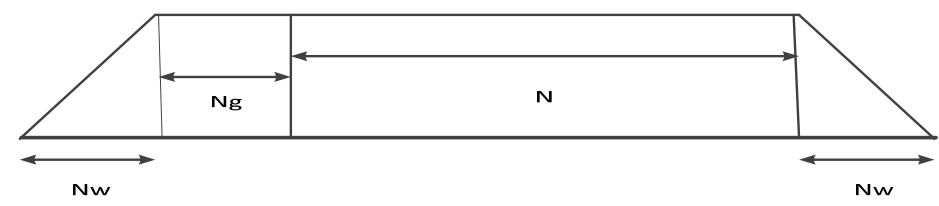

Fig.3 Structure of Windowed NC-OFDM Signal

\subsection{Filter Function Performance Analysis.}

The principle of selecting the filter function is to have better spectral characteristics, non-constant part cannot be too long to avoid the adjacent time domain signal have a greater impact. To maintain the orthogonality between subcarriers, Nyquist filter is usually used in NC-OFDM time domain filter. In this paper, five kinds of Nyquist filter are used, such as RC filter, BTRC filter, SOCW filter, Franks filter and Double-jump filter [7, 8].

We assume that the total number of subcarriers used by the NC-OFDM system is N, and the subcarriers range corresponding to $\mathrm{PU}$ is $[\mathrm{a}, \mathrm{b}]$. We define the average relative interference power as shown in formula (2). Where A is spectrum bandwidth and the average relative interference power is closely related to the waveform of the filter.

$$
\bar{P}_{R^{\rightarrow} L}=\frac{1}{\sqrt{A}} \int_{a}^{b}\left[\left|\sum_{n=0}^{a-1} a_{n} \frac{\sin \left(\pi\left(x-x_{n}\right)\right)}{\pi\left(x-x_{n}\right)}\right|^{2}+\left|\sum_{n=b+1}^{N-1} a_{n} \frac{\sin \left(\pi\left(x-x_{n}\right)\right)}{\pi\left(x-x_{n}\right)}\right|^{2}\right] d f
$$

\subsection{PTS Algorithm}

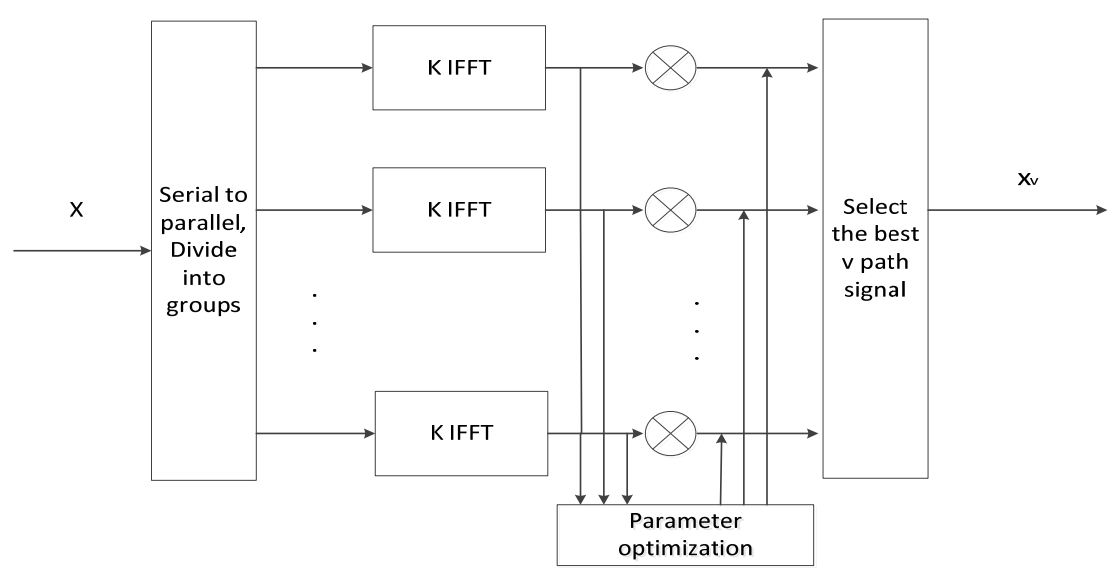

Fig.4 PTS-NC-OFDM Model

The idea of PTS algorithm is to group data and reduce PAPR by optimizing phase factor [7]. The PAPR of NC-OFDM signal is defined as formula (3). That is the ratio of the peak to average power of the signal. After serial to parallel, the signals are grouped, each group multiplied by different phase factors, and the lowest PAPR is chosen as the transmission signal. In the simulation of this paper, we choose the phase factor as $[\mathrm{pi} / 4, \mathrm{pi} / 2,3 \mathrm{pi} / 4, \mathrm{pi}]$.

$$
\operatorname{PAPR}(d B)=\frac{P_{\text {peak }}}{P_{\text {average }}}=10 \log _{10} \frac{\max \left\{|x(n)|^{2}\right\}}{E\left\{|x(n)|^{2}\right\}}
$$

We usually use CCDF (complementary cumulative distribution function) [8]to statistic the PAPR of NC-OFDM system. Where CCDF can be defined as: 


$$
P(P A P R>\lambda)=1-\left(1-\mathrm{e}^{-\lambda}\right)^{\alpha N}
$$

It is proved in article [9] that peak points can be captured enough when the sampling factor $J=4$, and the PAPR obtained is very close to its theoretical value. At this time, we usually take $\alpha=2.8$. In this paper, according to the suggestion of literature [8] that $\alpha$ is recommended as 2.8 .

\section{Simulations}

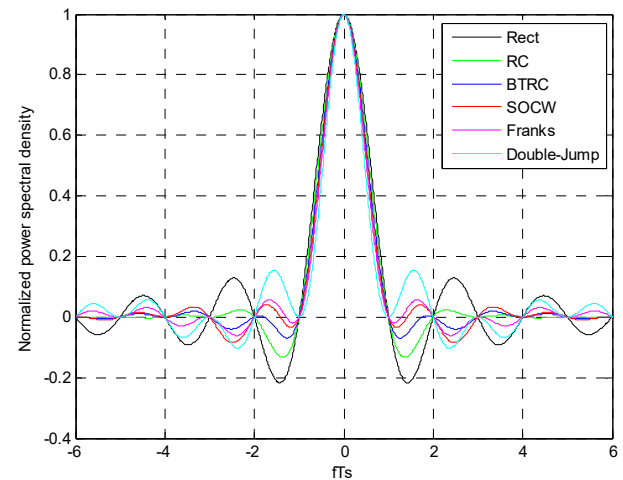

(a)

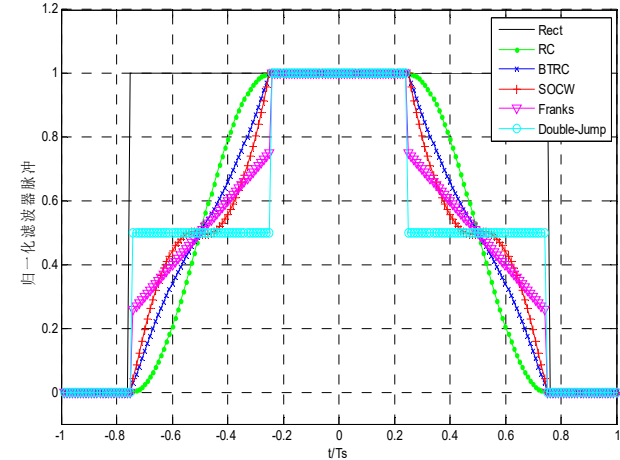

(b)

Fig. 5 Time and Frequency Domain Waveform of Filter Function

Fig 5 are respectively the time and frequency domain waveform for the five filter functions. It can be seen from Fig 5 that the power sidelobe of different filter functions in frequency domain is different.

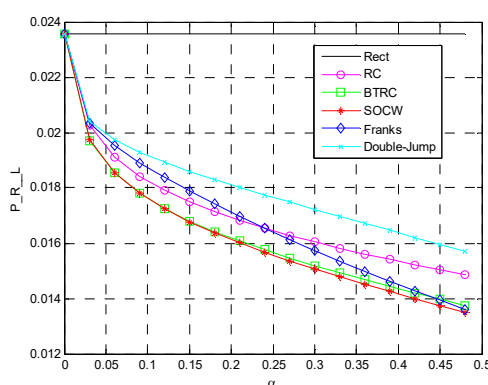

(a)

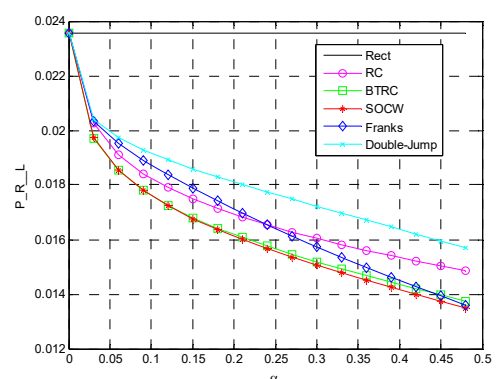

(b)

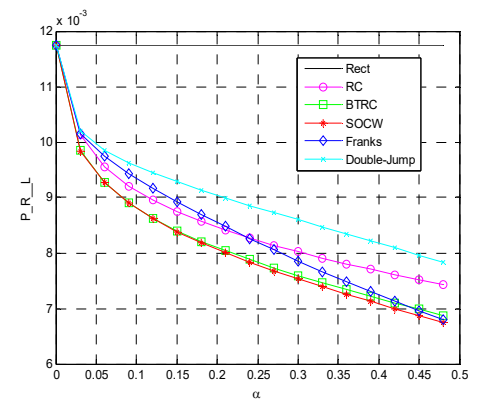

(c)

Fig. $6 P_{R^{\rightarrow} L}$ with Different Position

We set the total subcarriers $\mathrm{N}$ equal to 512 and the corresponding subcarriers of the PU are located at[10,137],[192,319],[376,503] respectively. The average relative interference power obtained by simulation is shown in Figure 6 (a), (b), (c). As you can see from Fig 6, the $P_{R^{\rightarrow} L}$ is relatively large when PU are in the middle position. When the PU are in the symmetrical position of the front end or the back end, the $P_{R^{\rightarrow} L}$ is equal. As it can be seen from the figure, with the increase of the roll-off factor $\alpha$, the average relative interference power $P_{R^{\rightarrow} L}$ is gradually becoming smaller. When $\alpha<0.23$, the average relative interference power of the five window functions is Double-Jump function, Franks function, RC function, BTRC function And SOCW function. While $\alpha \geq 0.23$, the $P_{R^{\rightarrow} L}$ of the five filter functions is Double-Jump function, RC function, Franks function, BTRC function and SOCW function. It can be seen from the figure that the SOCW window has the smallest average relative interference power regardless of the interval. 


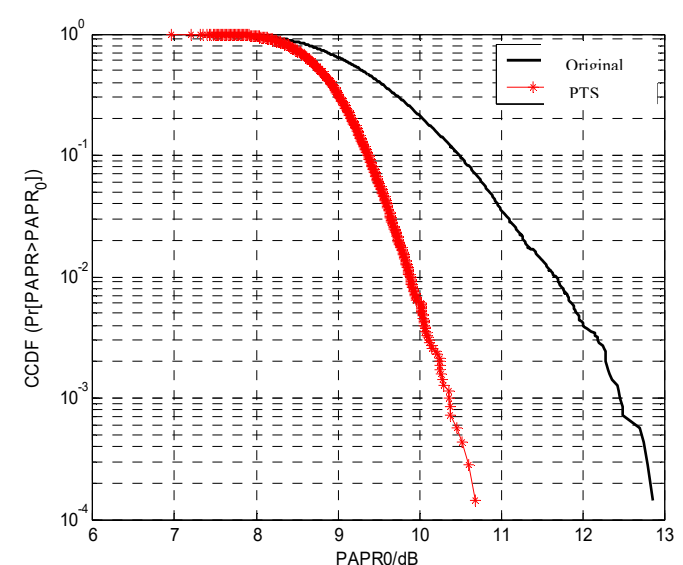

(a)

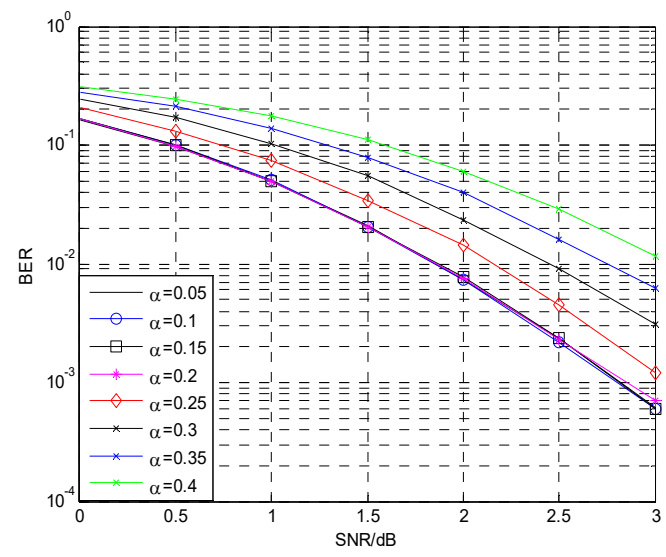

(b)

Fig. 7 Performance Analysis of PTS Algorithm after Adding Filter Function

It can be seen from figure 7 (a) that NC-OFDM algorithm can still use PTS algorithm to suppress PAPR after filtering. PAPR can be reduced by $2.2 \mathrm{~dB}$ under 0.001 probability. Combined with the better filter functions (SOCW and BTRC) in Figure 6, we get the BER of the receiver according to different roll off factors, as shown in Figure 7(b). It can be seen from Fig 6, when $\alpha>0.2$, with the roll-off coefficient $\alpha$ increases, BER value is also rising; when $\alpha \leq 0.2$, with the increase in $\alpha$, BER performance has almost no change.

\section{Summary}

In this paper, the power interference model of the authorized user is proposed based on the distribution of the subcarriers of the NC-OFDM system. We use the average relative interference power density $P_{R^{\rightarrow} L}$ to measure the different filter function that applied to the NC-OFDM system. The filter functions SOCW and BTRC has the lowest interference power among five filter functions. According to SOCW function, PAPR can be reduced by $2.2 \mathrm{~dB}$ under 0.001 probability and when $\alpha \leq 0.2$, with the increase in $\alpha$, BER performance has almost no change.

\section{References}

[1]. Martha Ann Stephen, Yamuna K. Moorthy. Sakuntala S. pilai. A Novel Method for joint PAPR reduction and Sidelobe Suppression in NC-OFDM Based on Cognitive Radio system[J]. IEEE Wireless Communication, 2016, 4, 1-6.

[2]. Rakesh Rajbansh, Alexander M. Peak-to-Average Power Ratio Analysis for NC-OFDM Transmissions. [J]. Vehicular Technology. 2007, 6, 18-24.

[3]. A Novel PAPR Reduction with Trellis shaping for NC-OFDM in Cognitive Radio [J]. Journal of Electronics (China), 2009, (03):296-302.

[4]. Martha Ann Stephen, Yamuna K. Moorthy. Sakuntala S. pilai. A Novel Method for joint PAPR reduction and Sidelobe Suppression in NC-OFDM Based on Cognitive Radio system[J]. IEEE Wireless Communication, 2016, 4, 1-6.

[5]. Mei Ling Li, a Hong Wang and Gang Fei Wang. An analysis Model for OFDMA based intercell interference. [J] Journal of Information Hiding and Multimedia Signal Processing .2016, 659-673.

[6]. Ashraf A. Eltholth. Probability Distribution Equalization Scheme for PAPR Reduction in NCOFDM Systems [J]. Networking and Mobile Computing, 2014, 6, 144-148. 
[7]. Guobin Zhang, Suili Feng. Subcarrier Allocation Algorithm Based on Graph-coloring in Cognitive Radio NC-OFDM System. [J]. Computer Science. 2011, 18, 24-31.

[8]. Rakesh Rajbansh, Alexander M. Peak-to-Average Power Ratio Analysis for NC-OFDM Transmissions. [J]. Vehicular Technology. 2007, 6, 18-24. 